BULLETIN Bulletin hispanique

HISPANIQUE Université Michel de Montaigne Bordeaux

$119-2 \mid 2017$

La Égloga renacentista en el Reino de Nápoles

\title{
Pedro Ruiz Pérez (ed.), Cervantes: los viajes y los días
}

Prosa Barroca y SIAL Ediciones, Madrid, 2016

\section{Álvaro Piquero Rodríguez}

\section{CpenEdition}

\section{Journals}

Edición electrónica

URL: http://journals.openedition.org/bulletinhispanique/5240

DOI: 10.4000/bulletinhispanique.5240

ISSN: 1775-3821

Editor

Presses universitaires de Bordeaux

\section{Edición impresa}

Fecha de publicación: 1 diciembre 2017

Paginación: 781-784

ISBN: 979-10-300-0218-8

ISSN: 0007-4640

Referencia electrónica

Álvaro Piquero Rodríguez, «Pedro Ruiz Pérez (ed.), Cervantes: los viajes y los días», Bulletin hispanique [En línea], 119-2 | 2017, Publicado el 28 noviembre 2017, consultado el 15 enero 2021. URL: http:// journals.openedition.org/bulletinhispanique/5240 ; DOl: https://doi.org/10.4000/bulletinhispanique. 5240

Este documento fue generado automáticamente el 15 enero 2021

Tous droits réservés 


\title{
Pedro Ruiz Pérez (ed.), Cervantes: los viajes y los días
}

Prosa Barroca y SIAL Ediciones, Madrid, 2016

\author{
Álvaro Piquero Rodríguez
}

\section{REFERENCIA}

Pedro Ruiz Pérez (ed.), Cervantes: los viajes y los días, Madrid: Prosa Barroca y SIAL

Ediciones, 2016, 267 págs.

1 Miguel de Cervantes Saavedra murió en Madrid en el mes de abril de 1616, y, como es habitual cuando se celebra una efeméride literaria de estas características, las actividades, estudios, exposiciones y ediciones del autor se multiplicaron cuantiosamente durante el año 2016, cuarto centenario de su muerte. Dentro de esa amplia nómina de publicaciones se incluye el volumen colectivo Cervantes: los viajes y los días, editado y coordinado por Pedro Ruiz Pérez.

El volumen, presentado en encuadernación rústica con solapas y una agradable maquetación que permite una cómoda lectura, está compuesto por doce artículos de extensión y tema variables. A pesar de ello, el libro no es una amalgama heterogénea de artículos que se van desgranando uno detrás de otro, sino que el editor ha buscado una ordenación lógica de todos ellos: los seis primeros están dedicados a la biografía de Cervantes o a la relación entre su vida y su obra, mientras que lo seis restantes centran su atención en la hermenéutica y en la búsqueda de nuevas interpretaciones de los textos cervantinos. Todos ellos, además, ofrecen al lector diferentes puntos de vista, exégesis complementarias y renovadas metodologías, demostrando que la crítica cervantina es un campo de estudio que está aún muy lejos de agotarse.

3 Más allá del breve preámbulo de Pedro Ruiz, el conjunto de artículos se abre con el estudio de Jorge García López, «Preliminares de una biografía científica». En este breve trabajo, de apenas diez páginas, el autor describe con rigor y acierto algunos puntos oscuros de la biografía de Cervantes, señalando los excesos interpretativos de una parte 
de la crítica. Ciertamente, la biografía del alcalaíno se ha centrado demasiadas veces en valoraciones subjetivas pseudoliterarias que no estaban respaldadas por ninguna documentación histórica. García López defiende la necesidad de ponderar la biografía científica y objetiva, basada en documentos, y alejarse definitivamente de las elucubraciones que buscan presentar a Cervantes como un mártir, un paradigma o un depravado sin más apoyo que el de la propia imaginación de los autores.

Las cuestiones relativas a la biografía de Cervantes continúan con Patricia Marín Cepeda y su artículo "“No soy bueno para palacio". Cervantes y el mecenazgo», donde se describen las distintas dedicatorias de las obras cervantinas y se intenta dilucidar cuáles consiguieron los réditos de mecenazgo que el autor buscaba con ellas. Tras este primer acercamiento puramente biográfico, Marín Cepeda reflexiona en la segunda parte del artículo sobre la originalidad de las dedicatorias al conde de Lemos, las únicas que parecen alcanzar su objetivo. Todo ello permite al lector hacerse una idea cabal de la importancia del mecenazgo en la época y comprender las razones de Cervantes para buscar incesantemente un protector a lo largo de su vida.

5 Al hilo de este tipo de análisis sociológicos, los tres artículos siguientes ahondan en las relaciones personales entre Cervantes y algunos autores contemporáneos. Ignacio García Aguilar apunta en su trabajo «Dos notas sobre la enemistad literaria entre Cervantes y Bernardo de la Vega» la posibilidad de que el segundo introdujera algunos ataques en clave hacia Cervantes en El pastor de Iberia. Ciertamente, las pruebas aportadas y el análisis de los pasajes clave son algo dudosos y enrevesados, por lo que resulta complicado entender ese cruce de elogios y personajes entre las obras de ambos autores en las que García Aguilar apoya su teoría. No obstante lo anterior, la hipótesis del investigador resulta muy sugerente $y$, uniendo todas las pruebas desgranadas, no se puede desechar la posibilidad de que esa enemistad existiera realmente.

6 Siguiendo esta misma línea, Pedro Ruiz Pérez analiza en «Cervantes y los poetas (I): ante el "Canto de Calíope"» las posibles relaciones de amistad entre Cervantes y otros autores a raíz de la galería de poetas que exhibe el autor en esta larga composición inserta en La Galatea. A través del análisis del texto y su comparación con la situación vital y social del autor en la época, Ruiz Pérez muestra muy afinadamente la intención real del poema. Además, distingue claramente entre los modelos literarios de Cervantes y los poetas nombrados con una mera intención social.

7 Este amplio estudio de Ruiz Pérez se ve complementado en buena medida por el trabajo de Adrián J. Sáez «Cervantes y el canon americano: el "Canto de Calíope", el Viaje del Parnaso y el "Discurso en loor de la poesía"", en el que, de manera similar a lo anterior, se analiza la relación entre Cervantes y los poetas del Nuevo Mundo a partir del análisis de la nómina de poetas americanos que aparece en los textos. Lamentablemente, la lista de autores nombrada por Cervantes es muy escasa, por lo que las conclusiones a las que puede llegar Sáez no son demasiado reveladoras. A pesar de ello, el estudio muestra algunos vínculos claros entre Cervantes y América y permite completar íntegramente el cuadro de relaciones literarias del autor alcalaíno.

8 Esta primera parte del volumen dedicada a la vida de Cervantes se cierra con el artículo de Javier Blasco «Avellaneda desde la estilometría». El estudio intenta dilucidar cuál puede ser el autor o los autores que se esconden detrás de Avellaneda a través de la aplicación de novedosas herramientas digitales como Stylo o JGAAP. Como el propio autor reconoce, las conclusiones a las que se puede llegar con este tipo de software no son en absoluto definitivas; no obstante, las posibilidades de análisis que ofrecen estas 
herramientas resultan enormemente útiles a la hora de abordar esta clase de investigaciones desde una perspectiva distinta y abarcadora. Sin duda, los estudios de autoría apoyados en herramientas digitales se van a multiplicar en un futuro no muy lejano, por lo que incluir un trabajo de estas características en el volumen era algo tan acertado como necesario.

9 La segunda parte del libro, centrada ya en la hermenéutica de la obra cervantina, comienza con un interesante acercamiento de Frederick A. de Armas a La Galatea y $\mathrm{El}$ Quijote desde un punto de vista pictórico. Como es habitual en los estudios interdisciplinares, Armas abre con su trabajo una nueva perspectiva de los textos cervantinos $\mathrm{y}$, desde el estudio de las técnicas pictóricas hasta la propia écfrasis, nos muestra un mundo literario plagado de referencias, recuerdos y comparaciones con algunos de los artistas más significativos del Renacimiento.

El heterogéneo conjunto de artículos de crítica literaria continúa con dos estudios que, aunque sea de manera tangencial, coinciden en el análisis: Cervantes busca continuamente la inversión, la ironía y la reflexión con respecto a los paradigmas de su época. Así, Ruth Fine reflexiona sobre la representación poco estereotipada de los judíos y la reinterpretación de algunos pasajes bíblicos en las obras cervantinas que parecen tener cierta inspiración en sus años de cautiverio, como Los baños de Argel, La gran sultana o «El amante liberal». Georges Güntert, por su parte, ahonda en el «misterio [...] escondido» de la Novelas ejemplares y reflexiona acerca de la novedad genérica que suponen, pues la ejemplaridad no apunta únicamente al sentido moral de las narraciones, sino también a su componente estético, esto es, a su originalidad. El estudioso, además, indaga en los fundamentos de la poética de las novelas y muestra el incisivo punto de vista que adopta Cervantes en algunas de ellas.

Dejando ya de lado las obras menores de Cervantes, el volumen continúa con dos majestuosos trabajos centrados en El Quijote. El primer de ellos, firmado por Alberto Blecua, recupera, con algunos cambios, la sección «Estructura del Quijote» incluido en el prólogo de su edición de El Quijote (2007). Bajo el título «Forma y sentido del Quijote» Blecua repasa nuevamente los problemas estructurales de la Primera parte y el cambio de tono de la Segunda. No obstante, en esta segunda redacción añade información complementaria, puntualiza algunas afirmaciones anteriores y redondea definitivamente un estudio imprescindible para comprender en su inmensidad la estructura externa e interna de la obra magna de Cervantes.

El siguiente trabajo, firmado por Pierre Darnis, continúa con la temática quijotesca, aunque en esta ocasión el cincel incide sobre un pasaje muy concreto de la Segunda parte: la aventura con los Duques aragoneses. El largo estudio, de más de cuarenta páginas, busca desmenuzar punto por punto las distintas burlas a las que son sometidos don Quijote y Sancho en su estancia con los duques desde una perspectiva absolutamente nueva. Según la hipótesis de Darnis, todo este largo pasaje puede interpretarse en clave satírica y, de hecho, podemos encontrar en él varios niveles de interpretación. Con ello, Cervantes estaría apuntando con sus dardos a diversos personajes, estamentos y autores de su época. Sin duda, las conclusiones del artículo son realmente evocadoras e invitan al lector a releer este conocido pasaje con renovada atención y una inédita mirada.

El volumen se cierra con el estudio de Anne J. Cruz «Cervantes y los feminismos». En él, la profesora Cruz presenta un interesante estado de la cuestión acerca de los estudios feministas dedicados a Cervantes desde los primeros testimonios, con Concha Espina, 
Carmen Castro y María Zambrano a la cabeza, hasta la actualidad. El trabajo, además, revisa los diferentes puntos de vista que el feminismo ha ido adoptando a lo largo de su historia en relación con la obra cervantina y, finalmente, apunta las carencias de las que aún adolece este tipo de análisis. Todo ello permite al lector hacerse una idea cabal de la evolución de los estudios de mujer dedicados a Cervantes de una manera clara e inmediata.

14 Con todo lo visto hasta aquí, queda claro que este volumen permite avanzar un paso más allá en el conocimiento de la vida y la obra de Miguel de Cervantes y demuestra que, cuatro siglos después de su muerte, los textos del autor aún tienen mucho que decir si se analizan desde un punto de vista novedoso y una metodología renovada.

\section{AUTORES}

\section{ÁLVARO PIQUERO RODRÍGUEZ}

Universidad Complutense de Madrid / Fundación Ramón Menéndez Pidal 